\title{
Homogeneous Intensity within the Rayleigh Length and Enhanced Depth of Focus for Gaussian Beams
}

\author{
Christian Bischoff ${ }^{* 1,2}$, Erwin Jäger ${ }^{1}$, Udo Umhofer ${ }^{1}$, Andrés Fabián Lasagni ${ }^{2,3}$ \\ and Friedemann Völklein ${ }^{4}$ \\ ${ }^{1}$ Topag Lasertechnik GmbH, Nieder-Ramstädter Str. 247, 64285 Darmstadt, Germany \\ ${ }^{2}$ Institut für Fertigungstechnik, Technische Universität Dresden, George-Bähr-Str. 3c, 01069 \\ Dresden, Germany \\ ${ }^{3}$ Fraunhofer-Institut für Werkstoff-und Strahltechnik IWS, Winterbergstr. 28, 01277 Dresden, \\ Germany \\ ${ }^{4}$ Institut für Mikrotechnologien, Hochschule RheinMain, Am Brückweg 26, 65428 Rüsselsheim, \\ Germany \\ *Corresponding author's e-mail: bischoff@topag.de
}

\begin{abstract}
The smallest width of a Gaussian beam at the focal plane is called waist and is only limited by the diffraction limit of the light. The waist determines the Rayleigh length which is the distance from the waist at which the width of the Gaussian beam increases by a factor of $2^{1 / 2}$ or the intensity decreases by a factor of 2 . With regard to laser material processing, these changes in spot size and intensity are typically undesirable. For example, variations in surface profiles or thickness of the processed material could require adjustment of focus. In this simulation study, we present a beam shaping method based on a development of the Fundamental Beam Mode Shaper (FBS) concept for enhanced depth of focus. Using this concept, an enhancement of Rayleigh length of $20 \%$ and a more homogeneous peak intensity around the focal plane is achieved.
\end{abstract}

DOI: $10.2961 /$ jlmn.2020.03.2004

Keywords: optical simulation, diffractive optic, phase shifting element, laser processing, beam shaping

\section{Introduction}

In the field of micro processing, pulsed solid-state laser are an indispensable tool meanwhile. Pulses with TEM00 Gaussian beam profiles, low energies with few $100 \mu \mathrm{J}$ and durations in the nanosecond (short pulse), pico-second or femtosecond (ultra-short pulse) range are typically provided by such systems. Actual research is carried out in particular in the field of ultra-short pulse systems, due the minimized laser induced damage [1].

It is known that a Gaussian distribution is the desired spatial intensity profile for many applications because it maintains its distribution during propagation and focusing. It enables an excellent focusing down to few micrometers, resulting in a high structural resolution of the laser micromachining process. The smallest width of a Gaussian beam at the focal plane, called waist which, is only limited by the diffraction limit of the light. The waist determines the Rayleigh length which is the distance from the waist at which the width of the Gaussian beam increases by a factor of $2^{1 / 2}$ or the intensity decreases by a factor of $2[2,3]$.

Since a certain laser fluence threshold is needed for the ablation during the laser processing, changes in spot size and thus in intensity and power density are typically undesirable. For very small spots in the $10 \mu \mathrm{m}$ range, this can only be a few $100 \mu \mathrm{m}$ in Rayleigh length, which limits the processing work area to this distance. High-precision processing, places additional demands on the inclination, flatness and irregularities of the samples. Axicons can be used to increase the depth of field and thus the process stability $[4,5]$. However, there are also several drawbacks to using axicons. On the one hand, they generally lead to low energy efficiency for this application. Constructive interference effects near the focal point lead to strong intensity modulations, which are distributed concentrically around the focus [6]. In addition to the energy loss, this can generate undesirable effects on the material and reduce the lateral resolution. Axicons with minimum size tip are difficult to manufacture. Manufacturing errors can lead to further interference effects along the optical axis, which also negatively affects the intensity of the extended focus [7]. For this reason, methods have recently been developed that combine an axicon with a binary phase plate, resulting in a significant reduction in intensity modulation $[8,9]$. Other concepts use spatial light modulators (SLM) to provide the phase distribution required to generate an extended depth of field [10]. Such SLMs are typically expensive and show a limited damage threshold. SLM phase-only beam shaping for an extended depth of field also leads to unwanted interference effects, concentrically distributed around the focus. The interference effects can be reduced by adding an additional amplitude modulation [11]. However, this reduces the overall efficiency.

But a Gaussian beam profile in the focal plane is not the most suitable profile for every application. If a Gaussian beam profile is used, the part of the beam with intensity below the ablation threshold does not contribute to the laser 
process. This energy part can be absorbed, leading to unwanted heating of the material. With a more homogeneous intensity distribution a larger part of pulse energy contributes to the ablation process, leading to less heating of the material and thus providing higher efficiency. Several studies have shown that the use of so-called Top-Hat beam profiles with constant level energy distribution can improve quality and efficiency of different laser processes [12, 13].

In this work, we focus on the so-called Fundamental Beam Mode Shaper (FBS) which originally was developed to generate a Top-Hat beam in the focal plane of a lens [14]. The FBS concept has been shown to be advantageous for Laser-Induced Breakdown Spectroscopy (LIBS) [15, 16], Matrix-assisted Laser Desorption/Ionization (MALDI) [17], Direct Laser Interference Patterning (DLIP) [18] and thin film applications $[19,20]$.

In order to gain a better understanding of the propagation behavior of an FBS shaped beam, detailed simulation study is carried out in this work. It is shown how, in addition to the homogenization of the intensity in the focus, a greater depth of field can be achieved with a simple binary FBS phase plate, only by introducing small changes in the optical setup. The optical element has high transmission and efficiency. The enhanced depth of focus shows no unwanted interference effects. By superimposing two FBS phase distributions a further enhancement of depth of focus is achieved.

\section{Important features of FBS concept}

The FBS concept is described in detail in [14]. The concept uses a binary phase information which is applied to a Gaussian input beam. The lateral structure of the phase information is given by the parameters $b$ and $s$ (see Fig. 1), and can be described by Eq. 1 .

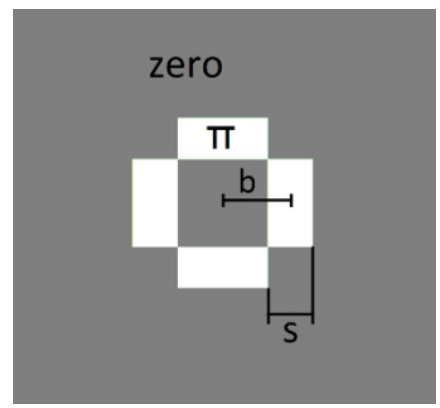

Fig. 1 Binary phase distribution of FBS concept with two phase values: $\pi \&$ zero

$$
\begin{aligned}
& \quad \operatorname{phase}=\pi \cdot\left(\operatorname{rect}\left(\frac{x \pm b}{s}\right) \cdot \operatorname{rect}\left(\frac{y}{2 b-s}\right)+\operatorname{rect}\left(\frac{y \pm b}{s}\right) .\right. \\
& \left.\operatorname{rect}\left(\frac{x}{2 b-s}\right)\right)
\end{aligned}
$$

This phase information can be converted into an optical component using lithographic methods like Reactive-IonEtching (RIE) and Ion-Beam-Etching (IBE) [21 - 23] leading to a FBS phase plate.

For the desired beam shaping configuration, a Gaussian beam propagates through a FBS phase plate and is focused by a lens. The amplitude $\mathrm{g}(\mathrm{x}, \mathrm{y})$ of a Gaussian beam is given by:

$$
g(x, y)=\exp \left(-\frac{x^{2}+y^{2}}{\omega^{2}}\right)
$$

defining $\omega$ as the radius of the Gaussian beam where the amplitude of the profile has dropped to $1 / \mathrm{e} \approx 0.368$

Originally the FBS concept was developed to generate a homogeneous Top-Hat profile in the focal plane of a lens. Based on a parameter scan and on the results shown in [14] the following requirements for the FBS phase structure must be fulfilled:

$$
\begin{aligned}
& s=b-\frac{s}{2} \\
& s=\omega
\end{aligned}
$$

The FBS phase plate shows only two levels. The height difference depends on the wavelength used. For optical glass with a refractive index of approximately 1.5 the height difference is in the same range as the wavelength. Typical radii of Gaussian input laser beams are in the range of millimeter. Due to equations 3 and 4 the lateral structure size $\mathrm{s}$ of the FBS phase plate is also in the millimeter range. This relatively large lateral structure does not place special requirements on well-established lithographic manufacturing processes. The lateral structure size and the height difference can be set and measured very precisely, smaller than $10 \mathrm{~nm}$, leading to negligible errors. A detailed description of the FBS manufacturing process is shown in [14].

In this work we show that by varying the FBS structure size $s$ the intensity of a Gaussian beam around the focal plane can be homogenized as well as the Rayleigh length will be increased.

\section{Simulation Model}

The FBS concept is based on diffraction of coherent light. For the simulation of the propagation behavior of focused beams, the commercial physical optics software VirtualLab Fusion was used, on a workstation with an AMD Ryzen ${ }^{\mathrm{TM}}$ Threadripper ${ }^{\mathrm{TM}}$ 2990WX processor, 32 cores, $3 \mathrm{GHz}$ and $128 \mathrm{~GB}$ RAM memory. VirtualLab Fusion provides two physical optics simulation engines, Classical Field Tracing and Field Tracing $2^{\text {nd }}$ Generation. In this study Field Tracing $2^{\text {nd }}$ Generation was used, which selects automatically the most suitable Fourier transform out of Fast Fourier Transformation, Semi-analytical Fourier Transformation and Homeomorphic Fourier Transformation [24, 25]. In Fig. 2 the used simulation strategy is shown. The parameter $\mathrm{z}$ indicates the absolute positions along the propagation axis $z$. The Gaussian input beam, the used FBS phase function as well as the focusing ideal lens are combined at position $\mathrm{z}=0$.

For a certain combination of Gaussian input beam radius $\omega$, lateral FBS structure size $s$ and focal length $f$ of ideal lens the intensity distribution along the propagation axis $\mathrm{z}$ was calculated in front and behind the focal plane.

Preliminary investigations have shown that distances between the planes of Gaussian input beam, FBS phase and lens of less than one meter do not have any significant influence on the intensity distribution along the caustic. Since the distances between the different planes would only unnecessarily increase the computing time, they were set to zero. Larger distances have not been investigated yet. 


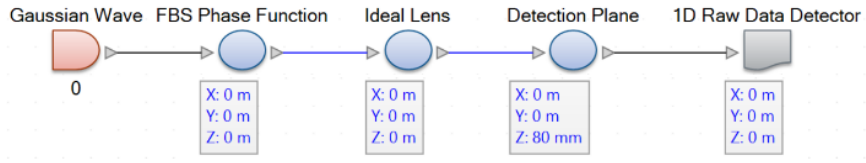

Fig. 2 Simulation strategy used in VirtualLab Fusion

\section{Simulation Results}

At first, the propagation behavior of a focused Gaussian beam is compared with the behavior of a FBS Top-Hat shaped beam. Some striking intensity profiles of FBS shaped beam along propagation axis $\mathrm{z}$ are identified. It will be shown how the FBS structure size $s$ can influence the location of these intensity profiles and how this could be used to homogenize the intensity along the optical axis around the focal plane.

\subsection{Caustic of FBS shaped beam}

In this section, images of the calculated caustic of focused beams are presented. To generate these images several cross-sections of the intensity profile along the $\mathrm{x}$-axis are calculated at different positions along the propagation axis $\mathrm{z}$ (optical axis) and stringed together. It is also assumed, that for each $\mathrm{z}$ position the cross-sections of the $\mathrm{y}-$ axis are equal to the cross-sections of the $\mathrm{x}$-axis. Each displayed image of a calculated caustic is normalized to the maximum intensity.

In Fig. 3 the caustic around the focal plane of an ideal focused Gaussian beam with wavelength $\lambda=532 \mathrm{~nm}$, focal length $\mathrm{f}=80 \mathrm{~mm}$ and input beam radius $\omega=2.5 \mathrm{~mm}$ is shown.

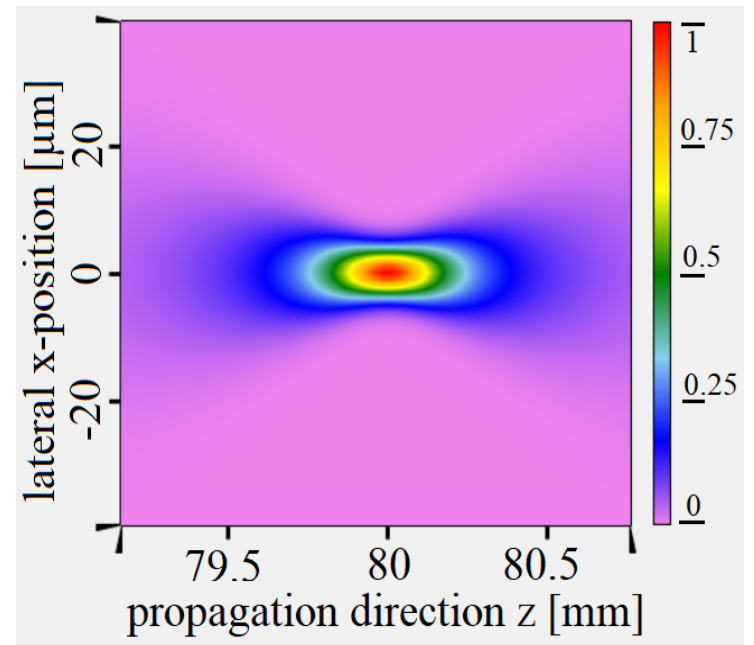

Fig. 3 Caustic of focused Gaussian beam along $\mathrm{z}$ axis

Taking a cross-section from Fig. 3, along $\mathrm{z}$ axis, with $\mathrm{x}=\mathrm{y}=0$ shows the variation of peak intensity along the optical axis for the propagating beam. As typical for focused Gaussian beams, the highest peak intensity is located in the focal plane at $80 \mathrm{~mm}$ (see Fig.4).

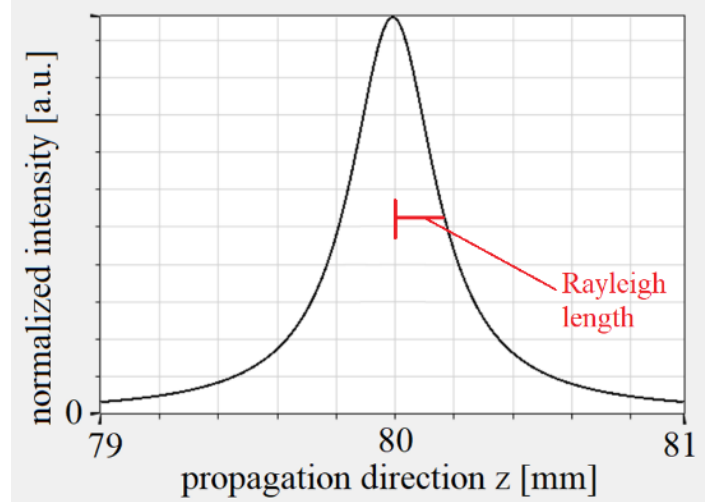

Fig. 4 Cross-section along optical axis $\mathrm{z}$ for focused Gaussian beam, $x=y=0$

The numerical aperture NA of the focused beam is calculated by:

$$
N A=\frac{\omega}{f}
$$

In the focal plane the beam shows the smallest width, called waist. The numerical aperture NA determines the waist $\omega_{\mathrm{f}}$ of a focused Gaussian beam with an ideal beam quality $\mathrm{M}^{2}=1$ :

$$
\omega_{f}=\frac{\lambda}{\pi \cdot N A}
$$

The waist $\omega_{\mathrm{f}}$ determines the Rayleigh length $\mathrm{z}_{\mathrm{r}}$ :

$$
z_{r}=\frac{\pi \cdot \omega_{f}^{2}}{\lambda}
$$

By applying the FBS phase to the Gaussian input beam and fulfilling the requirements according to Eq. 3 and 4, the caustic is changed as shown in Fig.5. A cross-section of Fig. 5, along the optical axis $\mathrm{z}$, with $\mathrm{x}=\mathrm{y}=0$ shows that the peak intensity on the optical axis varies. This variation of the peak intensity on the optical axis along the propagation axis $\mathrm{z}$ is shown in Fig. 6.

In addition, as shown in Fig. 5, five striking $\mathrm{x}, \mathrm{y}-$ intensity profiles could be detected along the propagation axis $\mathrm{z}$, consisting of three Top-Hat profiles with homogeneous cross-sections as well two peak intensity positions.

Beside the so-called zero order Top-Hat (zTH) in the focal plane two first order Top-Hats $( \pm 1 \mathrm{TH})$ are generated. One first order Top-Hat profile is in front and one behind the focal plane. Also, it could be seen that the highest peak intensity is not in the focal plane anymore. One of the highest peak intensities $( \pm \mathrm{PI})$ is in front and one behind focal plane. For the used NA=2.5/80 $=0.03125$ the locations are distributed symmetrically with respect to the focal plane. The location of $( \pm 1 \mathrm{TH})$ is roughly at 4 times the Rayleigh length from the focal plane. The location for the $( \pm \mathrm{PI})$ is close to 1.5 times the Rayleigh length from the focal plane. The plateau p shown in Fig.5 is the area with a constant homogeneous cross-section for the zero-order Top-Hat along beam direction. The normalized crosssections of the described intensity profiles as well as the corresponding unshaped Gaussian focal spot are shown in Fig.7. 


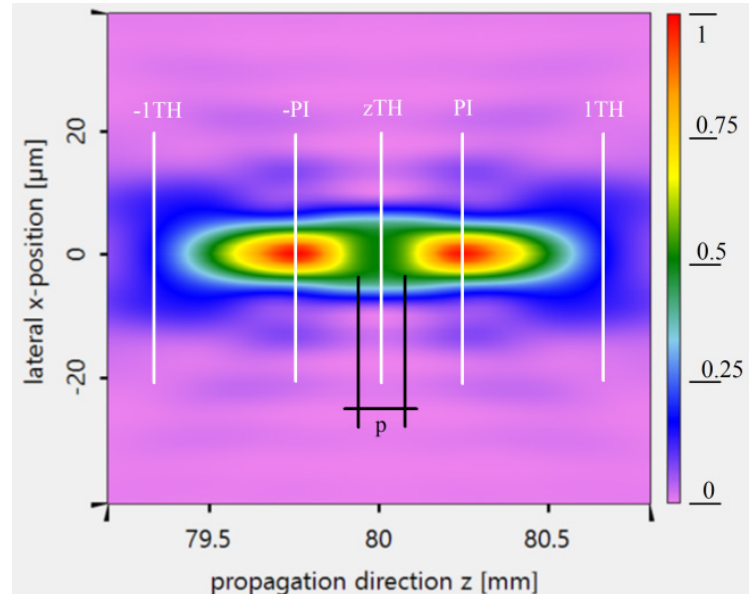

Fig. 5 Caustic of FBS shaped beam along $\mathrm{z}$ axis $(\mathrm{s}=\omega=2.5 \mathrm{~mm}, \mathrm{f}=80 \mathrm{~mm}, \mathrm{NA}=0.03125)$

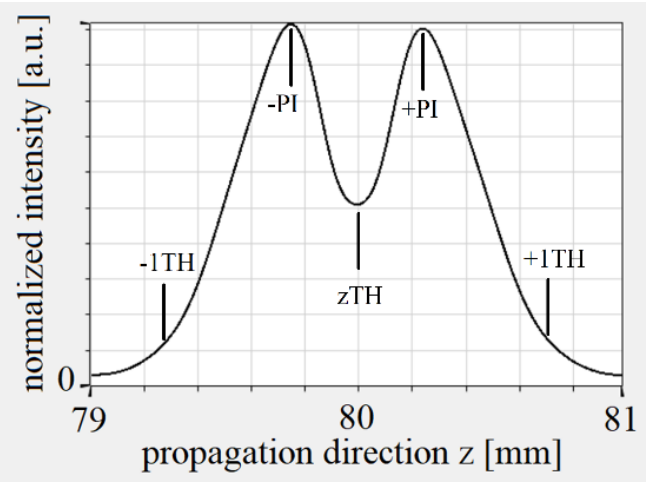

Fig. 6 Cross-section along optical axis $z(x=y=0)$ for FBS shaped beam according to Fig. 5 , relative intensity levels for important $\mathrm{z}$ positions are indicated
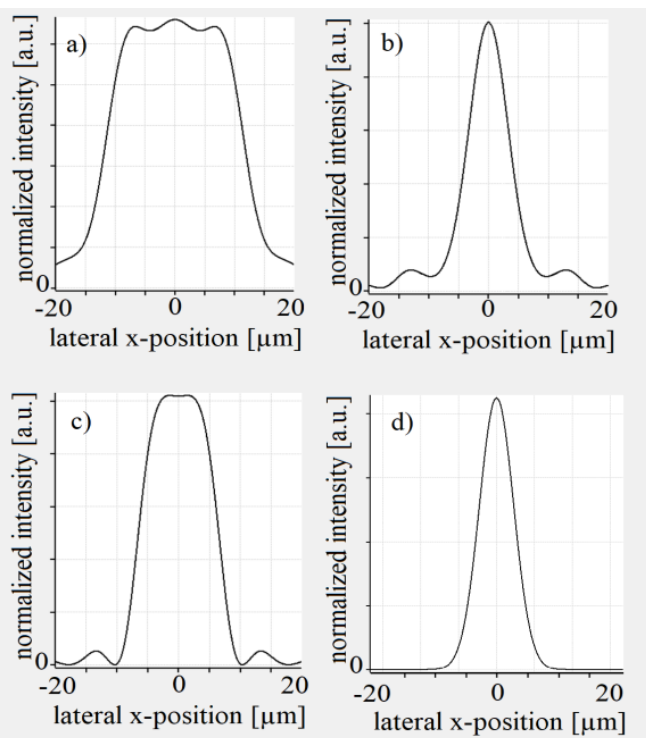

Fig. 7 Cross-section of striking intensity profiles, a) \pm first order Top-Hats $( \pm 1 \mathrm{TH}), \mathrm{b})$ peak intensities $( \pm \mathrm{PI})$,

c) zero-order Top-Hat (zTH) at focal plane, d) unshaped Gaussian focal spot

With $\mathrm{s}=\omega$ the spot size of $\mathrm{zTH}$ is $\sim 1.5$ times, the spot size of $( \pm 1 \mathrm{TH})$ is $\sim 3$ times larger than the unshaped Gaussian waist at the focal plane.

In addition to changing the light intensity near the optical axis, the FBS concept also creates side lobes around the desired spot profile. The maximum relative intensity of these side lobes with respect to the corresponding value on the optical is approximately $7 \%$ for the highest peak intensities $( \pm \mathrm{PI})$ and approximately $5 \%$ for the zero order TopHat (zTH). The relative intensity of the side lobes is influenced by the relation of the FBS phase structure size $\mathrm{s}$ to the Gaussian beam radius, as shown in the next sections.

\subsection{Influence of numerical aperture of focused beam}

When the NA of the focused beam is reduced, it can be seen that the symmetry of the propagation vanishes. In Fig. 8 the caustic of an FBS shaped beam is shown, with $\mathrm{s}=\omega=1$ and $\mathrm{f}=320 \mathrm{~mm}$ leading to $\mathrm{NA}=0.003125$. The $\max -$ imum value of the peak intensity (+PI) as well as the intensity of the first order Top-Hat $(+1 \mathrm{TH})$ behind the focal plane have lower values in comparison to the profiles in front of the focal plane. It means that for NA $\rightarrow 0$ the zeroorder Top-Hat is the far-field profile and every profile behind the focal plane vanishes. In addition, it can also be seen that the relative locations of $(+\mathrm{PI})$ and $(+1 \mathrm{TH})$ behind the focal plane are shifted. The maximum relative intensity of the side lobes remains approximately constant in comparison to the caustic with NA $=0.03125$ shown in Fig.5.

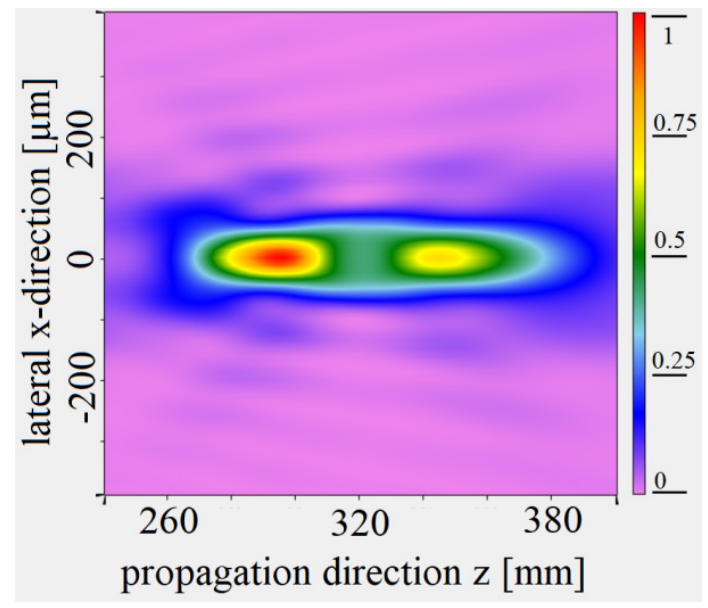

Fig. 8 Caustic of FBS shaped beam along $\mathrm{z}$ axis $(\mathrm{s}=\omega=1 \mathrm{~mm}, \mathrm{f}=320 \mathrm{~mm}, \mathrm{NA}=0.003125)$

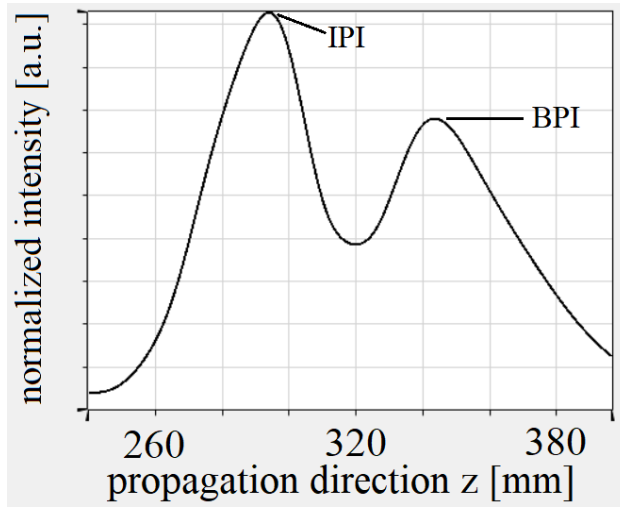

Fig. 9 Cross-section along optical axis $z(x=y=0)$ for FBS shaped beam according to Fig. 8, relative intensity levels for important $\mathrm{z}$ positions are indicated

In Fig. 9, the peak intensities for $( \pm \mathrm{PI})$ are indicated. By defining the maximum value of the peak intensity (-PI) in front of the focal plane as IPI, and the maximum value of the relative peak intensity (+PI) behind the focal plane as 
BPI, the homogeneity $h_{p}$ of both peaks can be calculated as follows:

$$
h_{p}=1-\frac{I P I-B P I}{I P I+B P I}
$$

Consequently, the homogeneity $h_{p}$ depends on the numerical aperture NA of the FBS shaped focused beam. This relation is shown in Fig. 10. For a homogeneity above 90\% the numerical aperture should be better than 0.005 .

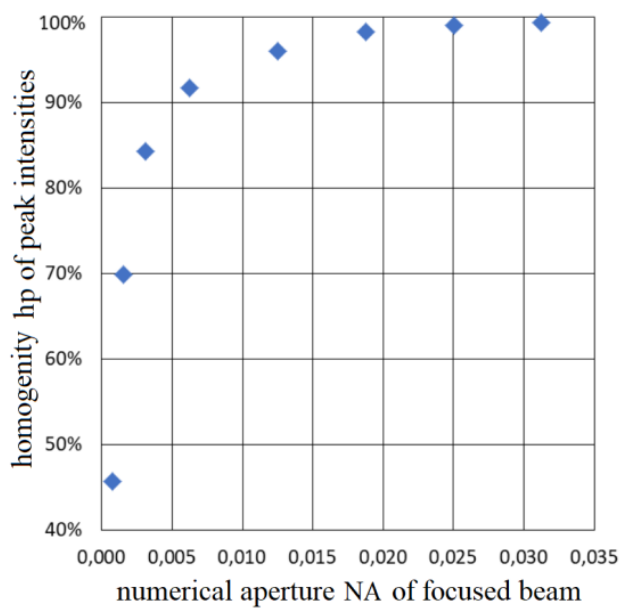

Fig. 10 Dependence of the homogeneity $h_{p}$ on the numerical aperture NA

The studies show that the homogeneity becomes better and better with increasing numerical aperture. NA values up to 0.1 were investigated and showed a homogeneity hp close to $100 \%$. The caustic as shown in Fig. 5 remains principally unchanged. Since the concept introduces only small phase changes, it can be assumed that higher values do not represent a problem either.

\subsection{Influence of FBS structure size}

When the dimensions of the FBS phase structure size $\mathrm{s}$ will be increased laterally with respect to the Gaussian beam, the diffraction effects will be reduced and the zeroorder Top-Hat vanishes. However, the location of the peak intensities (+/-PI) and the first-order Top Hat profiles (+/$1 \mathrm{TH})$ move towards the focal plane at the same time. This effect is shown in Fig. 11 using a FBS structure size increased by $25 \%(\mathrm{~s}=1.25 \omega)$ relative to the parameters in Fig. $5(\mathrm{~s}=1 \omega)$. With respect to Fig. 10 and $\mathrm{NA}=0.03125$ the homogeneity $h_{p}$ is close to $100 \%$ and we can state:

$$
I P I=B P I=P I
$$

By defining the intensity in the focal plane as FI the homogeneity $h_{f}$ of the intensity with respect to the peak intensity PI along the optical axis $\mathrm{z}$ around the focal plane can be calculated as given in Eq.10:

$$
h_{f}=1-\frac{P I-F I}{P I+F I}
$$

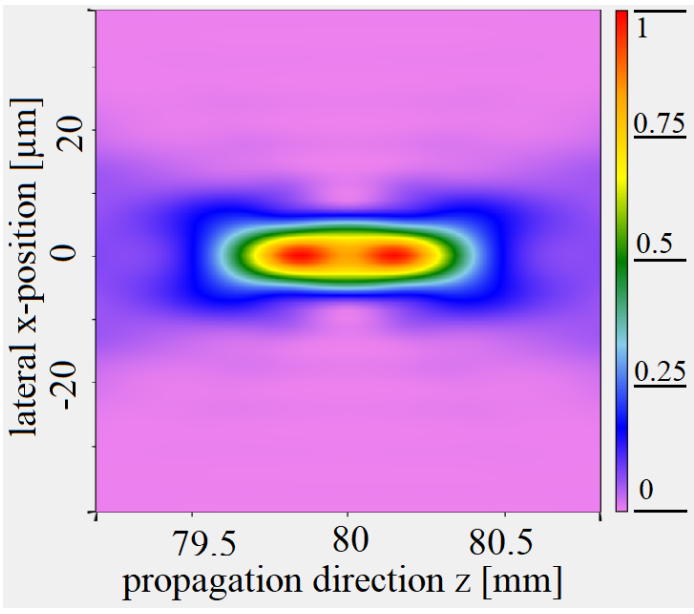

Fig. 11 Caustic of FBS shaped beam along $\mathrm{z}$ axis $(\mathrm{s}=1.25 \omega=3.125 \mathrm{~mm}, \mathrm{f}=80 \mathrm{~mm}, \mathrm{NA}=0.03125)$

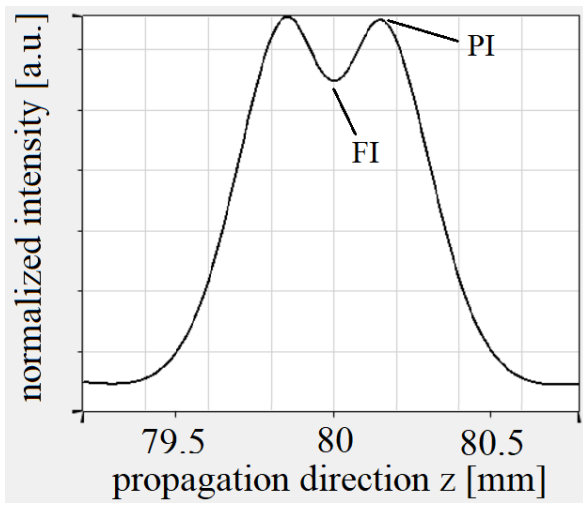

Fig. 12 Cross-section along optical axis $\mathrm{z}(\mathrm{x}=\mathrm{y}=0)$ for FBS shaped beam according to Fig. 11, relative intensity levels for important $\mathrm{z}$ positions are indicated

Due to the reduced diffraction effects the maximum relative intensity of the side lobes is also reduced. The intensity of the side lobes in the focal plane is approximately $1 \%$ and $3 \%$ for the highest peak intensities before and behind the focal plane, respectively.

\subsection{Connecting peak intensities}

As seen from Figs. 5 and 6 and Figs. 11 and 12 the FBS structure size $\mathrm{s}$ has an influence on the drop of the intensity FI in the focal plane and consequently on the homogeneity $\mathrm{h}_{\mathrm{f}}$. This relation is shown in Fig.13. It can be seen that there is no drop of intensity FI anymore when a FBS structure size $\mathrm{s}>1.5 \mathrm{\omega}$ is used. In this case the two peak intensities IPI and BPI can be merged, leading to $h_{f}=100 \%$.

For a focal length $\mathrm{f}=80 \mathrm{~mm}, \mathrm{~s}=3.75 \mathrm{~mm}$ and an input beam radius $\omega=2.5 \mathrm{~mm}(\mathrm{NA}=0.03125)$, the connected peak intensities are shown in Fig. 14.

Fig. 14 and 15 show an improvement of intensity homogeneity on optical axis along propagation direction in comparison to a Gaussian beam. But due to the diffraction effects introduced by the FBS phase information, the waist in the focal plane is slightly increased in comparison to the unshaped diffraction limited Gaussian focal spot. 


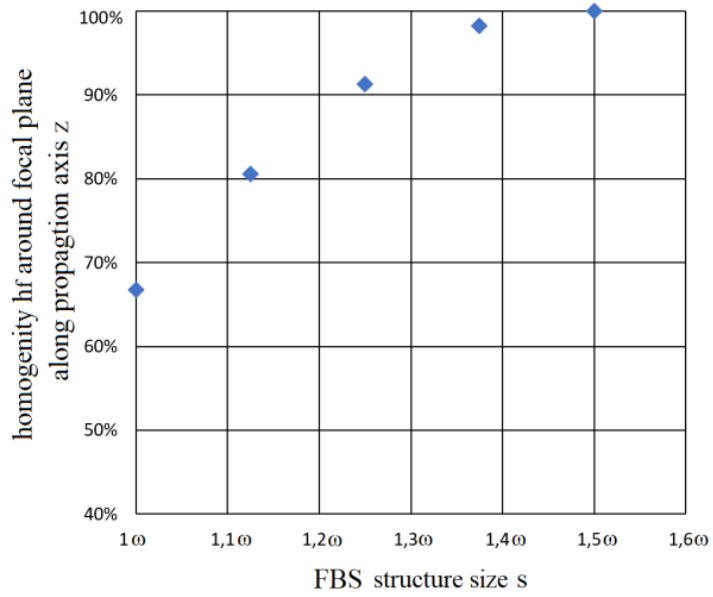

Fig. 13 Dependence of the homogeneity $h_{f}$ on the FBS structure size $\mathrm{s}$

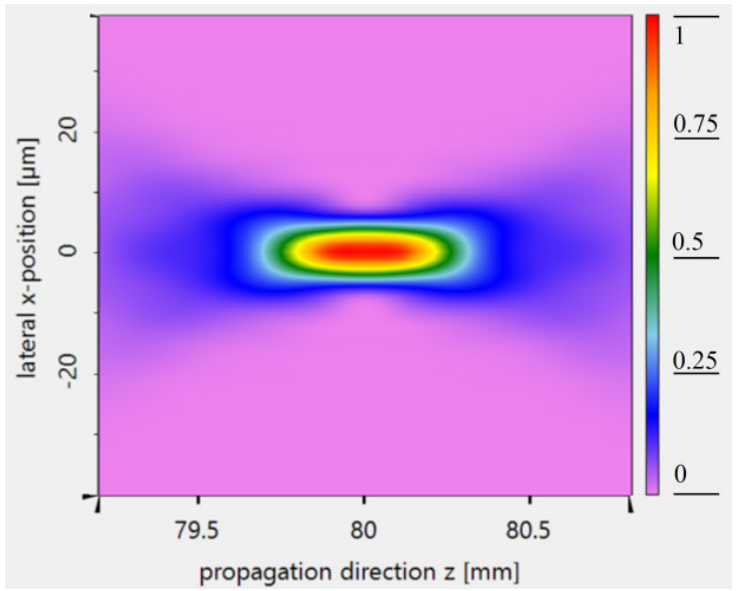

Fig. 14 Caustic of FBS shaped beam along $\mathrm{z}$ axis $(\mathrm{s}=3.75 \mathrm{~mm}, \omega=2.5 \mathrm{~mm}, \mathrm{f}=80 \mathrm{~mm}, \mathrm{NA}=0.03125)$

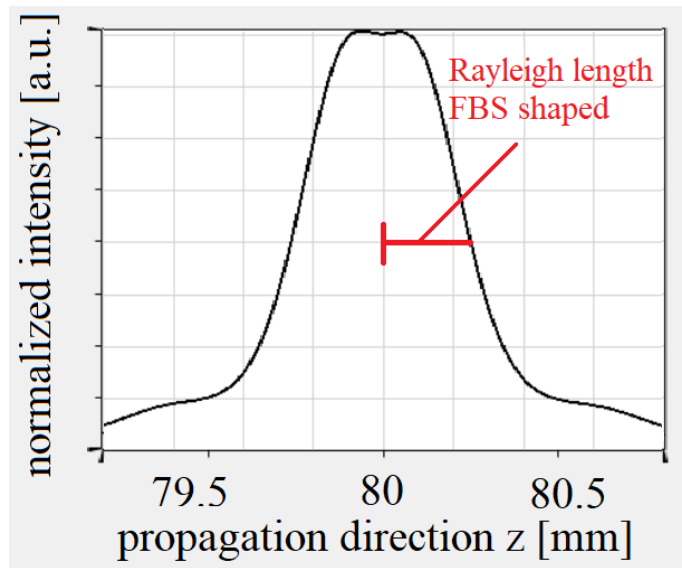

Fig. 15 Cross-section along optical axis $\mathrm{z}(\mathrm{x}=\mathrm{y}=0)$ for FBS shaped beam according to Fig. 14,

According to Eq. 7, the Rayleigh length depends on waist size. To make the Rayleigh length of unshaped and shaped beam comparable, the input Gaussian beam in the simulation for unshaped setup was slightly reduced. Table 1 shows the comparable results based on the same waist size $\omega_{\mathrm{f}}$ at the focal plane.
Table 1 Comparison of Rayleigh length $\mathrm{zr}_{\mathrm{r}}$

\begin{tabular}{ccccc}
\hline Input Beam & $\omega_{\mathrm{f}}[\mu \mathrm{m}]$ & $\omega[\mathrm{mm}]$ & $\mathrm{f}[\mathrm{mm}]$ & $\mathrm{Z}_{\mathrm{r}}[\mu \mathrm{m}]$ \\
\hline $\begin{array}{c}\text { Gaussian beam } \\
\begin{array}{c}\text { FBS } \\
\text { shaped beam* }\end{array}\end{array}$ & 5.97 & 2.27 & 80 & 210 \\
\hline$* \mathrm{~s}=1.5 \omega$ & 2.50 & 80 & 250 \\
\hline
\end{tabular}

By combining the Gaussian beam with a FBS phase (FBS structure size $\mathrm{s}=1.5 \omega$ ) the Rayleigh length could be increased by $\sim 20 \%$. Even more important is the fact that the profile shows constant peak intensity on optical axis along $30 \%$ of the improved Rayleigh length (see Figs. 14 and 15). At the same time the focal spot remains near Gaussian, showing a slight ellipticity of $\sim 1.04$, as shown in Fig. 16. The energy in the focus spot is $>99 \%$ of the input energy.

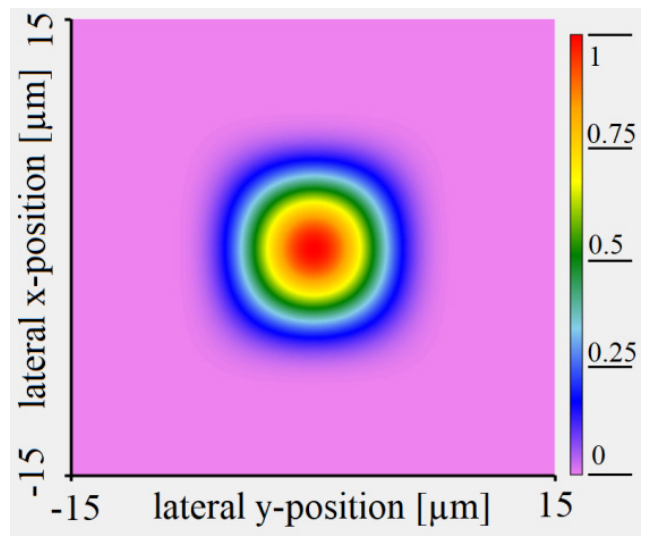

Fig. 16 Two-dimensional intensity distribution of FBS shaped beam at the focal plane, perpendicular to propagation axis $\mathrm{z}$

$(\mathrm{s}=3.75 \mathrm{~mm}, \omega=2.5 \mathrm{~mm}, \mathrm{f}=80 \mathrm{~mm}, \mathrm{NA}=0.03125)$

To switch the caustic of a FBS shaped beam between the Top-Hat and the Rayleigh application, the Gaussian input beam radius has to be increased by $50 \%$. This can easily be realized by placing the FBS phase plate on a motorized stage within beam expander, which allows a flexible use of such an element.

\subsection{Further enhancement of depth of focus}

As the beam at the focal plane in Fig. 16 remains Gaussian like while using one FBS phase, the procedure presented in 4.4 can be applied once more for a further enhancement of homogeneity and depth of focus.

Simulations showed that the Rayleigh length as well as the homogeneity along propagation axis could even be further enhanced, if an additional FBS phase with $\mathrm{s}_{2}=1.1 \omega$ is combined with the current setup. The resulting phase distribution is shown in Fig. 17. Two FBS phase structures, one with $\mathrm{s}_{1}=1.5 \mathrm{\omega}$ and a second with $\mathrm{s}_{2}=1.1 \omega$ are superimposed. This technique is referred to as double FBS shaping. By combining a Gaussian input beam with the double FBS phase (see Fig. 17) the caustic as shown in Fig. 18 will be realized. 


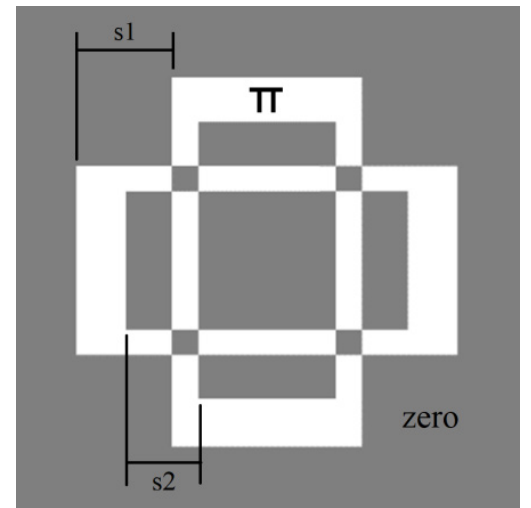

Fig. 17 Binary phase distribution realized by superimposing two FBS phase structures, one with $\mathrm{s}_{1}=1.5 \omega$ and a second with $\mathrm{s}_{2}=1.1 \omega$, double FBS shaped concept

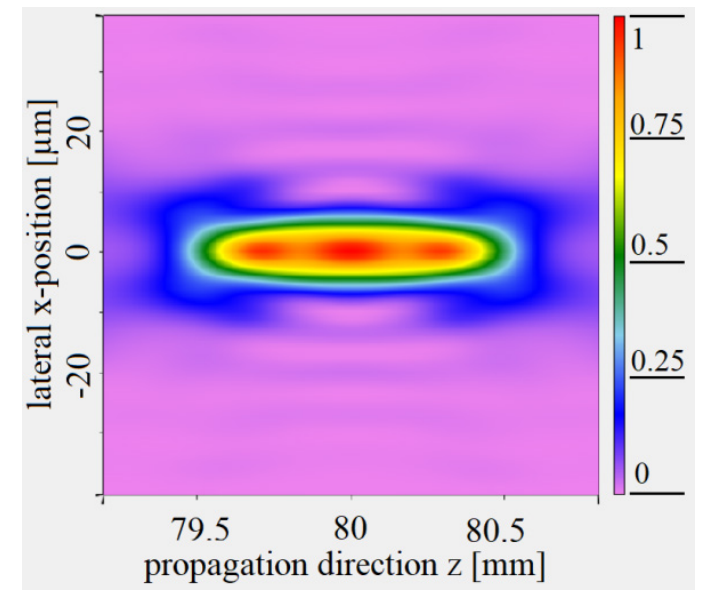

Fig. 18 Caustic of double FBS shaped beam along $\mathrm{z}$ axis $\left(\mathrm{s}_{1}=3.75 \mathrm{~mm}+\mathrm{s}_{2}=2.75 \mathrm{~mm} . \omega=2.5 \mathrm{~mm}, \mathrm{f}=80 \mathrm{~mm}, \mathrm{NA}=0.03125\right)$

Fig. 18 and 19 show the further improvement in homogeneity and depth of focus. In this case also diffraction effects lead to an enlargement of focus in comparison to the unshaped diffraction limited Gaussian focal spot.The maximum relative intensity of the side lobes in the focal plane increases to approximately $3 \%$ and to approximately $9 \%$ for the highest peak intensities before and behind the focal plane, respectively.

To make the Rayleigh length of unshaped and shaped beam comparable the input Gaussian beam in the simulation for unshaped setup was reduced. Table 2 shows the comparable results for the double FBS shaped concept based on the same waist size $\omega_{\mathrm{f}}$ in the focal plane.

Table 2 Comparison of Rayleigh length $\mathrm{z}_{\mathrm{r}}$

\begin{tabular}{ccccc}
\hline Input Beam & $\omega_{\mathrm{f}}[\mu \mathrm{m}]$ & $\omega[\mathrm{mm}]$ & $\mathrm{f}[\mathrm{mm}]$ & $\mathrm{Zr}_{\mathrm{r}}[\mu \mathrm{m}]$ \\
\hline Gaussian beam & 7,44 & 1,82 & 80 & 327 \\
$\begin{array}{c}\text { Double FBS } \\
\text { shaped beam* }\end{array}$ & 7,44 & 2.50 & 80 & 470 \\
\hline
\end{tabular}

$* \mathrm{~s}_{1}=1.5 \omega+\mathrm{s}_{2}=1.1 \omega$
From the table 2 it can be also seen that by using the double FBS shaping concept the Rayleigh length by a given spot size at the focal plane could be increased by $\sim 40 \%$ and the profile shows constant peak intensity on optical axis along $70 \%$ of the improved Rayleigh length (see also Fig. 19). The resulting two-dimensional intensity profile in the focal plane is shown in Fig. 20. The focal spot remains near Gaussian, showing some diffraction effects and a slight ellipticity of $\sim 1.04$. The energy in the focus spot is $>90 \%$ of the input energy.

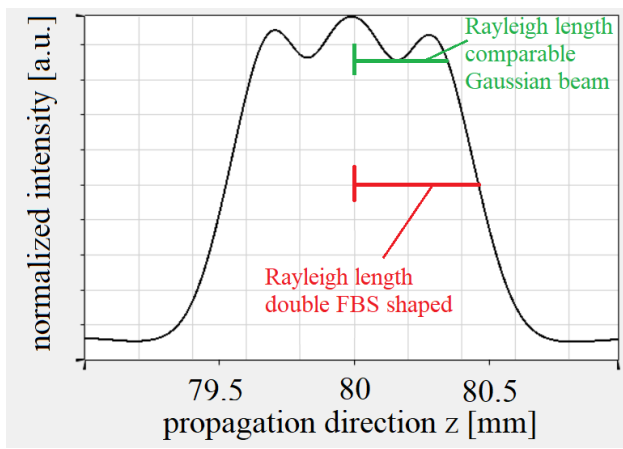

Fig. 19 Cross-section along optical axis $z(x=y=0)$ for double FBS shaped beam according to Fig. 18

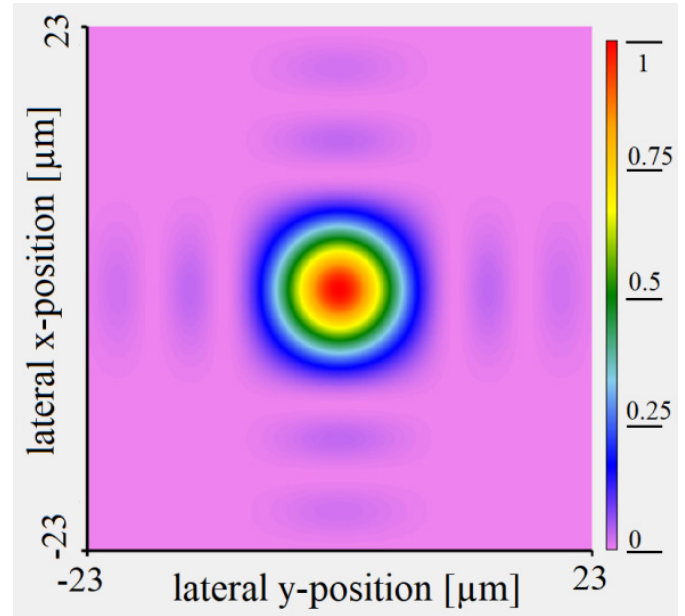

Fig. 20 Two-dimensional intensity distribution of double FBS shaped beam at the focal plane, perpendicular to propagation axis $\mathrm{z}\left(\mathrm{s}_{1}=3.75 \mathrm{~mm}+\mathrm{s}_{2}=2.75 \mathrm{~mm} . \omega=2.5 \mathrm{~mm}, \mathrm{f}=80 \mathrm{~mm}, \mathrm{NA}=0.03125\right)$

\section{Conclusion}

In this study we demonstrated how the FBS concept for generation of homogeneous spot profiles at a focal plane can also be used to overcome the limitations of the Rayleigh length. By varying the size of the Gaussian beam, which propagates through the optical element, the user can switch between both features. This can be realized by placing the optical element at different positions within the beam expander. Using the FBS concept with an adjusted input beam size an enhancement of Rayleigh length of $20 \%$ and a more homogeneous peak intensity around the focal plane is achieved. By superimposing two FBS phase distributions a further enhancement of the Rayleigh length up to $40 \%$ is possible. 


\section{Acknowledgments}

This work has been developed in the project "OpEn Laser Lathe" which is carried out as part of the European funding program "Eurostars" (E!12423). The German partners were funded by the Federal Ministry of Education and Research (FKZ:01QE1852A).

$$
\begin{aligned}
& \text { GEFÖRDERT VOM } \\
& \text { und Bundesministerium } \\
& \text { und Forschung }
\end{aligned}
$$

The Eurostars Programme is powered by EUREKA and the European Community

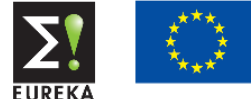

The work of A. F. Lasagni is supported by the German Research Foundation (DFG) under Excellence Initiative program by the German federal and state government to promote top-level research at German universities.

\section{References}

[1] B.N. Chichkov, C. Momma, S. Nolte, F. von Alvensleben, and A. Tünnermann: Appl. Phys. A, 63, (1996) 109.

[2] J. Alda: "Encyclopedia of Optical Engineering" ed. by R.G. Driggers (Publisher Marcel Dekker, Inc., 2003) 999.

[3] H. Urey: Appl. Opt., 43, (2004) 620.

[4] I. Alexeev, K.-H. Leitza, A. Otto, and M. Schmidt: Phys. Proc. 5, (2010) 533.

[5] X. Sun, H. Gao, B. Zeng, S. Xu, W. Liu, Y. Cheng, Z. $\mathrm{Xu}$, and G. Mu: Opt. Lett., 73, (2012) 857.

[6] M. Lei and B. Yao: Opt. Comm., 239, (2004) 367.

[7] P. Wu, C. Sui, and W. Huang: Photon. Res., 2, (2014) 82.

[8] A. S. Metel, M. M. Stebulyanin, S. V. Fedorov, and A. A. Okunkova: Technologies, 7, (2019) 5.

[9] F. He, J. Yu, Y. Tan, W. Chu, C. Zhou, Y. Cheng, and K. Sugioka: Sci. Rep., 7, (2017) 40785.
[10]D. Pan, S. Liu, S. Ji, Z. Cai, J. Li, Y. Hou, W. Zhang, S. Fan, R. Li, W. Zhu, D. Wu, and J. Chu: Opt. Lett., 45, (2020) 897.

[11]D. Pan, B. Xu, S. Liu, J. Li, Y. Hu, D. Wu, and J. Chu: Opt. Lett., 45, (2020) 2584.

[12] G. Di Domenico, G. Ruocco, C. Colosi, E. DelRe, and G. Antonacci: Sci. Rep., 8, (2018) 17178.

[13]H. Chuang and Y. Lee: J. Laser Micro/Nanoengin., 9, (2014) 198.

[14]C. Bischoff, F.Völklein, J. Schmitt, U. Rädel, U. Umhofer, E. Jäger, and A.F. Lasagni: Materials, 12, (2019) 2254

[15] J. Jia, H. Fu, Z. Hou, H. Wang, Z. Wang, F. Dong, Z. Ni, and Z. Zhang: Spectrochimica Acta Part B, 163, (2020) 105747.

[16]C. Basler, A. Brandenburg, K. Michalik, and D. Mory: Sensors, 19, (2019) 4133.

[17] M. Wiegelmann, K. Dreisewerd, and J. Soltwisch: J. Am. Soc. Mass Spectrom., 27, (2016) 1952.

[18] M. El-Khoury, B. Voisiat, T. Kunze, and A. F. Lasagni: J. Laser Micro/Nanoengin., 13, (2018) 268.

[19] R. Raciukaitis, E. Stankevicius, P. Gecys, M. Gedvilas, C. Bischoff, E. Jäger, U. Umhofer, and F. Völklein: J. Laser Micro/Nanoengin., 6, (2011) 37.

[20] S. Rung, M. Rexhepi, C. Bischoff, and R. Hellmann: J. Laser Micro/Nanoengin., 8, (2013) 309.

[21]R.E. Lee: "Ion-Beam Etching (Milling)", VLSI Electronics Microstructure Science, Volume 8, ed. by N.G. Einspruch and D.M. Brown, (Academic Press: Cambridge, MA, USA, 1984) 341.

[22] J. Schmitt, C. Bischoff, U. Rädel, M. Grau, U. Wallrabe, and F. Völklein: Proc. SPIE 9628, (2015) 96281P.

[23]P.W. Leech: Vacuum, 55, (1999) 191.

[24]Z. Wang, S. Zhang, O. Baladron-Zorita, C. Hellmann, and F. Wyrowski: Opt. Express, 27, (2019) 15335.

[25]Z. Wang, O. Baladron-Zorita, C. Hellmann, and F. Wyrowski: Opt. Express, 28, (2020) 10552.

(Received: June 26, 2020, Accepted: October 5, 2020) 